\title{
Absenteísmo de usuários como fator de desperdício nas diferentes modalidades de
}

\section{gestão na saúde}

Absenteeism of users as a waste factor in different modalities of health management

El absentismo de los usuarios como factor de desperdicio en diferentes modalidades de gestión sanitaria

\section{Resumo}

O absenteísmo de usuários é considerado um problema crônico mundial na assistência à saúde, tanto no setor público como no privado, levando ao desperdício de recursos. O objetivo do presente estudo foi estimar a taxa e o desperdício de recursos monetários vinculados ao absenteísmo de usuários em consultas e exames especializados, nas diferentes modalidades de gestão no Sistema Único de Saúde (SUS), na Região de Saúde Metropolitana do Espírito Santo (RSM-ES), nos anos de 2014 a 2016. Foram analisados 1.002 .719 procedimentos, sendo 666.182 consultas e 336.537 exames agendados nos 38 locais executantes, sendo 07 da administração direta e 31 da indireta. Os dados foram dos registros administrativos do Sistema de Regulação do Estado do Espírito Santo (SISREG-ES). Os valores monetários foram obtidos usando a Tabela SUS, tabela complementar de convênios e tabela de custos estimados, segundo o tipo de prestador. Para consultas, foram encontradas as maiores taxas e valores desperdiçados com o absenteísmo, no serviço privado $42,7 \%$ ( $\mathrm{R} \$ 2.000,00)$ e no público 39,1\% ( $\$ 2.255 .320,00)$. Para exames, no filantrópico 34,9\% (4.459.951,03) e no público com gestão OSS 32,4\% (R\$8.943.943,26). A quantidade de estabelecimentos, a produção e o volume de recursos ofertados pelo SUS para exames especializados se concentram na administração indireta, tendo o setor filantrópico e o público com gestão de OSS como principais provedores. As taxas e os valores desperdiçados nas diferentes modalidades de gestão evidenciam o desafio na agenda dos gestores do SUS para garantir a integralidade da atenção.

Palavras-chave: Absenteísmo; Atenção secundária à saúde; Recursos financeiros em saúde; Administração de serviços de saúde.

\begin{abstract}
Absenteeism is a chronic healthcare problem worldwide in both the public and private sectors, leading to waste of financial resources. To estimate the rate and the waste of monetary resources linked to patient absenteeism in consultations and specialized examinations on the management modalities of the Brazilian Unified Health System (SUS) in the Metropolitan Health Region of the state of Espirito Santo (RSMES), from 2014 to 2016 . A total of $1,002,719$ procedures were analyzed, of which 666,182 were medical consultations and 336,537 were examinations scheduled in one of the 38 executing sites, being 7 from direct administration and 31 from indirect administration. Data were obtained from the administrative records of the Regulation System of the State of Espirito Santo (SISREGED. Monetary values were obtained from the SUS table, a complementary table of contracts and a table of estimated costs according to the type of service provider. For medical consultations, the highest rates and values wasted with absenteeism were found, in the private service $42.7 \%(\mathrm{R} \$ 2,000.00)$ and in the public $39.1 \%$ ( $\mathrm{R} \$ 2,255,320.00)$. For exams, in philanthropic $34.9 \%(\mathrm{R} \$ 4,459,951.03)$ and in the public with OSS management 32,4\% (R\$ 8,943,943.26) The amount of healthcare units, the production and the number of resources provided by the SUS for specialized
\end{abstract}


examinations were mostly destined to indirect administrations, mainly the philanthropist and the public with health social organization providers. The rates of absenteeism and the wasted resources evidence the challenge in the SUS manager's agenda.

Keywords: Absenteeism; Secondary care; Financial resources in health; Health services administration.

\section{Resumen}

El absentismo es un problema sanitario crónico en todo el mundo, tanto en el sector público como en el privado, que genera un derroche de recursos económicos. Objetivo: Estimar la tasa y el derroche de recursos monetarios vinculados al ausentismo de los pacientes en las consultas y exámenes especializados sobre las modalidades de gestión del Sistema Único de Salud (SUS) de Brasil en la Región Metropolitana de Salud del estado de Espirito Santo (RSMES), de 2014 a 2016. Métodos: Se analizaron un total de 1.002 .719 procedimientos, de los cuales 666.182 fueron consultas médicas y 336.537 reconocimientos programados en uno de los 38 sitios de ejecución, siendo 7 de administración directa y 31 de administración indirecta. registros del Sistema de Regulación del Estado de Espirito Santo (SISREGED. Los valores monetarios se obtuvieron de la tabla SUS, una tabla complementaria de contratos y una tabla de costos estimados según el tipo de prestador de servicios. Resultados: Para consultas médicas, se encontraron las mayores tasas y valores desperdiciados con absentismo, en el servicio privado 42,7\% ( $\$$ \$2.000,00) y en el público $39,1 \%$ ( $\mathrm{R} \$ 2.255 .320,00)$. Por exámenes, en filantrópicos 34,9\% ( $\$$ \$ 4.459.951,03) y en público con gestión de OSS $32,4 \%$ ( R \$ 8.943.943,26). Conclusión: El monto de las unidades de salud, la producción y la cantidad de recursos que el SUS proporciona para exámenes especializados en su mayoría estaban destinados a administraciones indirectas, principalmente al filántropo y al público con proveedores de organizaciones sociales de salud. Las tasas de absentismo y el desperdicio de recursos evidencian el desafío en la agenda del gerente del SUS.

Palabras clave: Absentismo; Atención Secundaria de Salud; Recursos Financieros en Salud; Administración de los Servicios de Salud.

\section{Introdução}

O Sistema Único de Saúde (SUS) do Brasil se configura como um dos maiores sistemas universais do mundo. Entretanto, desde a sua existência, sofre com a concorrência do setor privado e enfrenta constrangimentos que afetam a capacidade do Estado em garantir o direito universal à saúde (Giovanella et al., 2018; Araújo et al., 2021). Na gestão do sistema, alguns desafios ameaçam a diretriz da integralidade. Entre estes, está o absenteísmo de usuários, que contribui de maneira significativa para restrição do acesso e é considerado um problema crônico na assistência à saúde, tanto no setor público como no privado, no Brasil e no mundo (Bender, Molina, \& Mello, 2010; Cavalcanti, Cavalcanti, Serrano, \& Santana, 2013a; Ellis \& Jenkins, 2012; Giunta et al., 2013; Jabalera Mesa, Morales Asencio, \& Rivas Ruiz, 2015; Kheirkhah, Feng, Travis, Tavakoli-Tabasi, \& Sharafkhaneh, 2015).

Considerando como um tema mundial relevante, Dantas, Fleck, Cyrino Oliveira, e Hamacher (2018), em revisão sistemática, identificaram que a taxa média de não comparecimento (absenteísmo) é de 23\%, sendo as maiores encontradas na África (43,0\%), América do Sul (27,8\%), Ásia (25,1\%), América do Norte (23,5\%), Europa (19,3\%) e Oceania (13,2\%). No SUS, tem-se revelado como um problema frequente em diversos tipos de atendimentos e especialidades médicas (Oleskovicz, Oliva, Grisi, Lima, \& Custódio, 2014), podendo atingir percentuais altos (Bittar et al., 2016), com taxas próximas ou superior a $25 \%$ (Oleskovicz et al., 2014).

A demanda na atenção especializada (AE) tem sido cada vez mais crescente, em razão do envelhecimento da população e do maior acesso aos serviços de saúde, o que impõe novos desafios e novas prioridades ao sistema (Barreto et al., 2011; Schmidt et al., 2011). O cuidado especializado é um dos maiores desafios do SUS, sendo necessário para dar efetividade e consequência à atenção básica e prover assistência integral aos que dela necessitam (Tesser \& Poli Neto, 2017).

Os estabelecimentos de saúde exibem diferentes modalidades com algumas composições organizacionais, formatos de administração e modelos de gestão (Secchi, 2009), podendo gerir, manter e prestar serviços de forma direta e indireta (Brasil, 2010). Na administração direta, as atividades gerenciais são desempenhadas pelo próprio Estado, através de sujeitos e órgãos que o integram e na administração indireta ele delega a atribuição de gerir a entes personalizados que, mantendo o vínculo estatal, prestam serviços públicos ou de interesse público (Ibañez \& Vecina Neto, 2007). Assim, para suprir suas necessidades, 
o SUS conta com a participação da gestão indireta para ampliar sua oferta de assistência, principalmente na atenção AE.

$\mathrm{Na}$ legislação brasileira, a participação complementar da gestão indireta na saúde se efetua por meio de contratos administrativos, convênios e contratos de gestão, quando há necessidade comprovada desta cooperação, mediante a impossibilidade de ampliação dos serviços próprios (Brasil, 2010), possibilitando também, complementar o valor da Tabela SUS, ao prestador, com recursos próprios sem prejudicar o acesso (Brasil, 2001).

Sob esses aspectos, é possível verificar que o SUS vem legalmente atrelado à participação do setor privado ao longo de sua existência, o que gera dificuldades de criar e manter um modelo de gestão pública que assegure a prestação de serviços à população sem depender da complementação do setor privado (Romano \& Scatena, 2014).

Segundo Paim (2009), o setor privado está visivelmente paralelo ao SUS, em várias situações, deixando o sistema de serviços de saúde no Brasil complexo e segmentado, comprometendo o acesso, a qualidade e a integralidade da atenção. A tendência à privatização é um problema ainda não resolvido, considerando que na atualidade não tem condições de ampliar sua rede de serviços próprios, para substituir os serviços executados pelo setor privado na sua cobertura (Ocké-Reis, 2016).

No Brasil, são poucas as publicações científicas que avaliam as modalidades de gestão de serviços de saúde. Em uma revisão narrativa sobre o tema, Ravioli, Soárez, e Scheffer, (2018), concluiram que as publicações são restritas e concentradas no estado de São Paulo, sendo inconclusivas quanto à superioridade de um modelo sobre o outro, deixando uma importante lacuna sobre as características, o funcionamento e a eficácia dos arranjos e modelos em curso no SUS. Na Região de Saúde Metropolitana do Espírito Santo (RSM-ES), o absenteísmo na AE é um problema frequente, verificado pela Secretaria Estadual de Saúde (SESA-ES), e tem ocasionado prejuízos à população e aos cofres públicos, visto que muitos pacientes agendados não comparecem e não desmarcam. Segundo os dados, no primeiro semestre de 2015, a taxa foi de 38\% para consultas e exames especializados agendados pela Central de Regulação (Rebello, 2015).

Diante dessas considerações, é possível propor que o absenteísmo seja uma fonte de desperdícios de recursos, e a gestão deve conhecê-lo, quantificando-o, por meio de custos de procedimentos não realizados (Bittar, Magalhães, Felizola, \& Falcão, 2016). Assim, o objetivo desta pesquisa foi estimar o valor monetário desperdiçado com absenteísmo em consultas e exames especializados, nas diferentes modalidades de gestão na RSM-ES.

\section{Metodologia}

Trata-se de um estudo descritivo baseado em dados administrativos da RSM-ES, no Estado do Espírito Santo, localizado na Região Sudeste do Brasil, sendo o estado menor e menos populoso da região. É composta por 20 municípios, incluindo a capital do estado, apresenta os melhores Índices de Desenvolvimento Humano Municipal (IDH-M) do estado, variando de 0,68 a 0,856, com população estimada para 2018 de 2.219 .349 habitantes de acordo com o Instituto Brasileiro de Geografia e Estatística (IBGE).

Apresenta diferenças no número populacional entre os municípios, sendo que em seis a população de residentes está em torno de 15 mil habitantes e, em quatro, é maior que 300 mil habitantes. Concentra 55,87\% de toda a população do estado, segundo dados do IBGE. Dos cinco municípios litorâneos, 98,7\% são de população urbana. A oferta de serviços especializados, está concentrada na capital e nos municípios próximos. Alguns mais distantes da capital, em torno de 153,90Km, apresentam serviços limitados à atenção básica. A região apresenta desigualdades entre os municípios em relação aos serviços de saúde (Secretaria Estadual de Saúde [SESA], 2011).

Os 38 locais executantes de serviços regulados de consultas e exames especializados na RSM-ES estão assim distribuídos: 07 serviços públicos da administração direta - 06 estadual e 01 federal e 31 serviços da administração indireta, sendo 20 privados, 03 públicos com gestão de OSS e 08 filantrópicos. Ao analisar a localização dos serviços, identificamos 


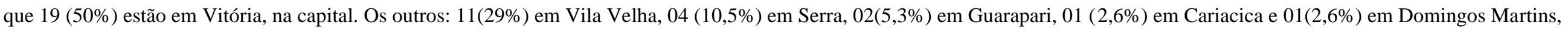

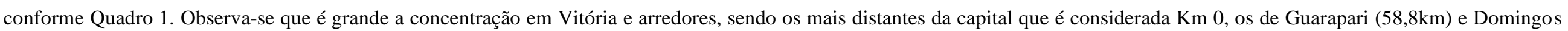
Martins $(52,2 \mathrm{~km})$.

Quadro 1 - Número e localização dos locais executantes por modalidade de gestão, na RSM-ES de 2014-2016.

\begin{tabular}{|c|c|c|c|c|c|c|c|}
\hline $\begin{array}{c}\text { Serviços por modalidade } \\
\text { de gestão }\end{array}$ & Vitória & Vila Velha & Serra & Guarapari & Cariacica & $\begin{array}{c}\text { Domingos } \\
\text { Martins }\end{array}$ & Total por modalidade \\
\hline Distância em km de Vitória & $\mathbf{0}$ & 7 & 23,4 & $\mathbf{5 8 , 8}$ & 29,8 & 52,2 & - \\
\hline \multicolumn{8}{|c|}{ GESTÃO DIRETA } \\
\hline Serviços Públicos & 3 & 2 & 1 & - & 1 & - & 07 \\
\hline \multicolumn{8}{|c|}{ GESTÃO INDIRETA } \\
\hline $\begin{array}{l}\text { Serviços Públicos com } \\
\text { gestão OSS }\end{array}$ & 2 & & 1 & - & - & - & $\mathbf{0 3}$ \\
\hline $\begin{array}{l}\text { Serviços } \\
\text { filantrópicos }\end{array}$ & 4 & 2 & - & 1 & - & 1 & 08 \\
\hline Serviços privados & 10 & 7 & 2 & 1 & - & - & 20 \\
\hline TOTAL POR MUNICÍPIO & 19 & 11 & 4 & 2 & 1 & 1 & 38 \\
\hline
\end{tabular}

Fonte Dados: SESA/PDR/ SISREG- Autores. 
Foram utilizados todos os dados dos agendamentos realizados pelo SISREG-ES fornecidos pela SESA-ES, no período de janeiro a dezembro de 2014 a 2016, num total de 1.002 .719 procedimentos, sendo 666.182 consultas e 336.537exames especializados. Os agendamentos referem-se às vagas disponibilizadas por especialidades pela Programação Pactuada Integrada (PPI), realizada entre estado e os municípios da região. Os diferentes locais executantes, participaram de maneiras variadas por especialidades. As variáveis selecionadas foram: ano (2014, 2015 e 2016), procedimento agendado (consulta e exame), procedimento confirmado (realizado), procedimento não confirmado (absenteísmo- não realizado), consultas e exames especializados (rol de procedimentos da AE) e local executante (local de procedimento agendado). Inicialmente os dados foram separados em consultas, exames e ano. Para as consultas, diante das diversas nominações usadas pelo SISREG-ES, foi necessário utilizar a Classificação Brasileira de Ocupações (CBO), que é uma norma de classificação numerativa e descritiva de atividades profissionais, para recodificar a especialidade médica e outras do profissional executante. Para a mesma especialidade haviam diversas nominações que foram agrupadas em uma única classificação da CBO para posterior codificação e definição de correspondência (de-para), com os procedimentos das tabelas de valores/preços utilizados.

Foram agrupadas por especialidades para cada local executante, quantificando-se o número de consultas agendadas, consultas confirmadas, absenteísmo e o montante financeiro por especialidade por ano. Posteriormente foram reagrupadas nas especialidades, por ano e por modalidade de gestão. Os exames especializados, estavam codificados pela tabela do SISREG-ES sendo necessário recodificá-los pela Tabela Unificada de Procedimentos, Medicamentos e Insumos Estratégicos do SUS (Tabela SUS), usando o mesmo procedimento adotado para as consultas. Após, foi inserido o preço individual dos exames de acordo com a modalidade de gestão igual nas consultas. Após, os exames foram agrupados para cada local executante e reagrupados por modalidade de gestão.

Os valores de cada procedimento variaram segundo remunerações vinculadas aos locais executantes e respectivas naturezas administrativas. Para os realizados nos estabelecimentos públicos estadual e federal (gestão direta) e privados (gestão indireta), a referência foi a Tabela SUS, ressaltando que, no serviço público federal, também foi utilizada tabela complementar de convênio. Nos serviços filantrópicos, o valor total foi a somatória da Tabela SUS e tabela complementar. Para os serviços públicos com gestão de OSS, foi utilizada tabela de custos estimados por local executante, fornecida pela SESA-ES e elaborada utilizando um software desenvolvido pela empresa de consultoria nacional Planisa, que adota a ferramenta denominada de Key performance Indicators for health (KPIH). Todos os valores considerados nos cálculos, foram atualizados para 2018. Os dados monetários foram estimados em um comparativo de valores dos procedimentos usando somente a Tabela SUS como parâmetro de preço para todas as modalidades de gestão e também utilizando para valor final a somatória da Tabela SUS e tabela complementar para os hospitais público federal, filantrópicos e com gestão de OSS.

A taxa de absenteísmo para consultas e exames especializados, em cada modalidade de gestão de 2014-2016, foi calculada com a divisão do total de procedimentos não confirmados (absenteísmo) (numerador) pelo total de procedimentos agendados (denominador), multiplicando-se o resultado por 100. O percentual de procedimento agendado para exames e consultas especializados em cada modalidade de gestão de 2014-2016, foi calculado com a divisão do total de procedimentos agendados para a modalidade (numerador) pelo número total de agendamentos (denominador) nas diversas modalidades, multiplicando-se o resultado por 100. Os valores monetários estimados para desperdício foram obtidos mediante a multiplicação do total de procedimentos não confirmados (absenteísmo) anualmente, por modalidade de gestão, pelos valores atribuídos a cada procedimento segundo a natureza administrativa do local executante. Para as análises foi utilizado o Programa Microsoft Office Excel 2010 e os dados foram apresentados de acordo com o objetivo da análise. O estudo foi autorizado pela SESA-ES por meio do Temo de Anuência de 26/9/2017 processo no 79619819/2017 e aprovado pelo Comitê de Ética em Pesquisa da UFES, respeitando a resolução do Conselho Nacional de Saúde em 2/5/2018, protocolo no 2.631.695. 


\section{Resultados e Discussão}

Foram contabilizados 666.182 procedimentos de consultas especializadas e 336.537 de exames especializados, agendados nos diversos locais executantes, agregados em quatro modalidades de gestão. Para as consultas, a taxa média de absenteísmo no período, foi de 38,6\% (257.025), e um desperdício estimado de R \$ 3.558.837,88. Para os exames especializados, a taxa média foi de 32,1\% (108.103) com um desperdício estimado de $\mathrm{R} \$ 15.007 .624,15$ (Tabela 1).

Nas consultas especializadas, as taxas de absenteísmo por modalidade de gestão variaram de $35,5 \%$ (serviço filantrópico) a 42,7\% (serviço privado). A menor taxa foi de 35,5\%, no filantrópico, com um total estimado de desperdício de $\mathrm{R} \$ 341.750,00$. O serviço público ficou com a segunda menor taxa de 39,1\%, num total de $\mathrm{R} \$ 2.255 .320,00$, seguido do público com gestão de OSS, com taxa de 39,6\%, e um total de R $\$ 959.767,88$. A maior taxa, 42,7\% foi do privado, num total de $\mathrm{R} \$ 2.000,00$. Entre os valores monetários desperdiçados atribuídos nas diferentes modalidades de gestão, o menor foi encontrado no serviço privado ( $\mathrm{R} \$ 2.000,00$ ), seguido do filantrópico ( $\mathrm{R} \$ 341.750,00)$ e do público com gestão de OSS ( $\mathrm{R} \$$ 959.767,88). O maior valor foi no público ( $\mathrm{R} \$ 2.255 .320,00$ ). Entretanto, vale destacar que $82,8 \%$ dos procedimentos de consultas foram agendados no serviço público.

Considerando o total dos serviços de consultas especializadas agendadas nos 38 locais executantes, a taxa de oferta foi de $(0,1 \%)$ para o serviço privado, seguida pelo serviço público com gestão de OSS de $(2,7 \%)$ e pelo serviço filantrópico $(14,4 \%)$. A maior oferta de agendamento de consultas especializadas foi de $(82,8 \%)$ no serviço próprio (Tabela 1$)$. Nos exames especializados, as taxas de absenteísmo por modalidade de gestão variaram de $26,1 \%$ a $34,9 \%$. A menor taxa $(26,1 \%)$ foi atribuída ao privado, num total de $\mathrm{R} \$ 781.687,87$, seguido de $28,5 \%$ do público, num total de $\mathrm{R} \$ 822.041,99$. Para o público com gestão de OSS, a taxa foi de 36,9\%, num total de $\mathrm{R} \$ 8.943 .943,26$. A maior taxa $(37,7 \%)$ ocorreu no serviço filantrópico, com um total de $\mathrm{R} \$ 4.896 .346,95$ (Tabela 1 ).

Quanto à taxa de oferta de exames especializados agendados, a menor foi 8,9\% no privado, seguida do público, com 16,5\%. No público com gestão de OSS, foi de 36,9\%. A maior oferta de agendamento ocorreu no serviço filantrópico - 37,7\%. (Tabela1). Identificou-se que, em consultas, usando a Tabela SUS, o valor desperdiçado foi de $\mathrm{R} \$ 2.570 .250,00$, no entanto, os mesmos procedimentos, se usadas a Tabela SUS e as tabelas de valores complementares diferenciadas, o desperdício foi de R $\$$ 3.556.837,88, perfazendo um valor monetário 38,4\% maior. Para os exames, o valor desperdiçado usando a Tabela SUS foi de $\mathrm{R} \$$ 6.677.140,16, no entanto, ao adicionarem-se as tabelas complementares, o desperdício foi de $\mathrm{R} \$ 15.007 .624,15$, perfazendo um aumento de $124,8 \%$ (Tabela 2). 
Tabela 1 -Procedimentos de consultas e exames com respectivas, taxas de serviço e de absenteísmo por modalidade de gestão e valores

\begin{tabular}{|c|c|c|c|c|c|c|c|c|c|c|c|c|c|c|c|c|c|}
\hline \multicolumn{18}{|c|}{ CONSULTAS } \\
\hline & \multirow{2}{*}{\multicolumn{4}{|c|}{2014}} & \multirow{2}{*}{\multicolumn{4}{|c|}{2015}} & \multirow{2}{*}{\multicolumn{4}{|c|}{2016}} & \multicolumn{5}{|c|}{ 2014-2016 } \\
\hline & & & & & & & & & & & & & \multicolumn{2}{|c|}{ Agendamento } & \multicolumn{3}{|c|}{ Desperdício } \\
\hline & $\mathbf{A}$ & C & Abs. & Valor R\$ & $\mathbf{A}$ & C & Abs. & Valor & $\mathbf{A}$ & C & Abs. & Valor & Total & $\%$ & $\mathbf{N}$ & $\mathbf{R} \$$ & $\%$ \\
\hline $\begin{array}{c}\text { Serviço } \\
\text { Filantrópico }\end{array}$ & 30.039 & 19.922 & 10.117 & $101.170,00$ & 31.773 & 20.305 & 11.468 & $114.680,00$ & 34.316 & 21.726 & 12.590 & $125.900,00$ & 96.128 & 14,4 & 34.175 & $341.750,00$ & 35,5 \\
\hline $\begin{array}{l}\text { Serviço } \\
\text { Público* }\end{array}$ & 160.389 & 100.376 & 60,013 & $624.010,00$ & 189.587 & 115.493 & 74.094 & $775.100,00$ & 201.444 & 120.097 & 81.347 & $856.210,00$ & 551.420 & 82,8 & 215.454 & $2.255 .320,00$ & 39,1 \\
\hline $\begin{array}{c}\text { Serviço } \\
\text { Público/ } \\
\text { Gestão OSS }\end{array}$ & 10.186 & 6.293 & 3.893 & $537.768,18$ & 4.358 & 2.466 & 1.892 & $243.076,33$ & 3.622 & 2.211 & 1.411 & $178.923,37$ & 18.166 & 2,7 & 7.196 & $959.767,88$ & 39,6 \\
\hline $\begin{array}{c}\text { Serviços } \\
\text { Privados }\end{array}$ & 217 & 114 & 103 & $1.030,00$ & 100 & 62 & 38 & 380,00 & 151 & 92 & 59 & 590,00 & 468 & 0,1 & 200 & $2.000,00$ & 42,7 \\
\hline Total & 200.831 & 126.705 & 74.126 & $1.262 .948,18$ & 225.818 & 138.326 & 87.492 & $1.132 .856,33$ & 239.533 & 144.126 & 95.407 & 1.161.033,37 & 666.182 & 100,0 & 257.025 & $3.558 .837,8$ & 38,6 \\
\hline \multicolumn{18}{|c|}{ EXAMES } \\
\hline $\begin{array}{l}\text { Serviços } \\
\text { Privados }\end{array}$ & 9.092 & 6.642 & 2.450 & $248.247,53$ & 11.626 & 8.380 & 3.246 & 293.976,24 & 9.295 & 7.167 & 2.128 & $239.464,10$ & 30.013 & 8,9 & 7.824 & $781.687,87$ & 26,1 \\
\hline $\begin{array}{c}\text { Serviço } \\
\text { Público* }\end{array}$ & 17.766 & 12.786 & 4.480 & 237.196,87 & 17.717 & 12.946 & 4.771 & $245.404,45$ & 20.126 & 14.031 & 6.095 & $339.440,67$ & 55.609 & 16,5 & 15.846 & $822.041,99$ & 28,5 \\
\hline $\begin{array}{c}\text { Serviço } \\
\text { Público/ } \\
\text { Gestão OSS }\end{array}$ & 36.365 & 22.235 & 14.130 & $2.214 .626,66$ & 42.038 & 28.432 & 13.606 & $3.180 .120,06$ & 45.685 & 33.159 & 12.526 & $3.549 .196,54$ & 124.088 & 36,9 & 40.262 & $8.943 .943,26$ & 32,4 \\
\hline $\begin{array}{c}\text { Serviço } \\
\text { Filantrópico }\end{array}$ & 41.029 & 26.816 & 14.213 & 1.457.028,48 & 42.978 & 27.237 & 15.741 & $1.504 .614,09$ & 42.820 & 28.603 & 14.217 & $\mathbf{1 . 4 9 8 . 3 0 8 , 4 6}$ & 126.827 & 37,7 & 44.171 & $4.459 .951,03$ & 34,9 \\
\hline Total & 104.252 & 68.479 & 35.773 & $4.157 .099,54$ & 114.359 & 76.995 & 37.364 & $5.224 .114,84$ & 117.926 & 82.960 & 34.966 & $5.626 .409,77$ & 336.537 & 100,0 & 108.103 & $15.007 .624,15$ & 32,1 \\
\hline
\end{tabular}

A: Agendada / C: Confirmada / Abs: Absenteísmo / N: Absenteísmo total. Fonte Dados: SESA/ SISREG- Elaboração própria do autor. 
Tabela 2 - Procedimentos de consultas e exames com respectivas taxas de serviço e de absenteísmo, por modalidade de gestão e valores da Tabela SUS.

\begin{tabular}{|c|c|c|c|c|c|c|c|c|c|c|c|c|c|c|c|c|c|}
\hline \multicolumn{18}{|c|}{ CONSULTAS } \\
\hline & \multirow{2}{*}{\multicolumn{4}{|c|}{2014}} & \multirow{2}{*}{\multicolumn{4}{|c|}{2015}} & \multirow{2}{*}{\multicolumn{4}{|c|}{2016}} & \multicolumn{5}{|c|}{ 2014-2016 } \\
\hline & & & & & & & & & & & & & \multicolumn{2}{|c|}{ Agendamento } & \multicolumn{3}{|c|}{ Desperdício } \\
\hline & $\mathbf{A}$ & $\mathbf{C}$ & Abs. & Valor R\$ & $\mathbf{A}$ & C & Abs. & Valor R\$ & $\mathbf{A}$ & C & Abs. & Valor R\$ & Total & $\%$ & $\mathbf{N}$ & Valor R\$ & $\%$ \\
\hline $\begin{array}{c}\text { Serviço } \\
\text { Filantrópico }\end{array}$ & 30.039 & 19.922 & 10.117 & $101.170,00$ & 31.773 & 20.305 & 11.468 & $114.680,00$ & 34.316 & 21.726 & 12.590 & $125.900,00$ & 96.128 & 14,4 & 34.175 & $341.750,00$ & 35,5 \\
\hline $\begin{array}{l}\text { Serviço } \\
\text { Público }\end{array}$ & 160.389 & 100.376 & 60.013 & $600.130,00$ & 189.587 & 115.493 & 74.094 & $740.940,00$ & 201.444 & 120.097 & 81.347 & $813.470,00$ & 551.420 & 82,8 & 215.454 & $2.154 .540,00$ & 39,1 \\
\hline $\begin{array}{c}\text { Serviço } \\
\text { Público/ } \\
\text { Gestão OSS }\end{array}$ & 10.186 & 6.293 & 3.893 & $38.930,00$ & 4.358 & 2.466 & 1.892 & $18.920,00$ & 3.622 & 2.211 & 1.411 & $14.110,00$ & 18.166 & 2,7 & 7.196 & $71.960,00$ & 39,6 \\
\hline $\begin{array}{l}\text { Serviços } \\
\text { Privados }\end{array}$ & 217 & 114 & 103 & $1.030,00$ & 100 & 62 & 38 & 380,00 & 151 & 92 & 59 & 590,00 & 468 & 0,1 & 200 & $2.000,00$ & 42,7 \\
\hline Total & 200.831 & 126.705 & 74.126 & $741.260,00$ & 225.818 & 138.326 & 87.492 & $874.920,00$ & 239.533 & 144.126 & 95.407 & $954.070,00$ & 666.182 & 100,0 & 257.025 & $2.570 .250,00$ & 38,6 \\
\hline \multicolumn{18}{|c|}{ EXAMES } \\
\hline $\begin{array}{l}\text { Serviços } \\
\text { Privados }\end{array}$ & 9.092 & 6.642 & 2.450 & 248.247,53 & 11.626 & 8.380 & 3.246 & 293.976,24 & 9.295 & 7.167 & 2.128 & 239.464,10 & 30.013 & 8,9 & 7.824 & $781.687,87$ & 26,1 \\
\hline $\begin{array}{l}\text { Serviço } \\
\text { Público }\end{array}$ & 17.766 & 12.786 & 4.480 & 232.787,98 & 17.717 & 12.946 & 4.771 & 236.279,56 & 20.126 & 14.031 & 6.095 & 332.030,41 & 55.609 & 16,5 & 15.846 & $385.646,07$ & 28,5 \\
\hline $\begin{array}{c}\text { Serviço } \\
\text { Público/Gestão } \\
\text { OSS }\end{array}$ & 36.365 & 22.235 & 14.130 & $592.520,68$ & 42.038 & 28.432 & 13.606 & $802.728,95$ & 45.685 & 33.159 & 12.526 & $975.504,18$ & 124.088 & 36,9 & 40.262 & $2.370 .753,81$ & 32,4 \\
\hline $\begin{array}{c}\text { Serviço } \\
\text { Filantrópico }\end{array}$ & 41.029 & 26.816 & 14.213 & 987.331,07 & 42.978 & 27.237 & 15.741 & $884.458,42$ & 42.820 & 28.603 & 14.217 & $851.811,05$ & 126.827 & 37,7 & 44.171 & $3.139 .052,41$ & 34,9 \\
\hline Total & 104.252 & 68.479 & 35.773 & $2.060 .887,26$ & 114.359 & 76.995 & 37.364 & 2.217.443,17 & 117.926 & 82.960 & 34.966 & $2.398 .809,73$ & 336.537 & 100,0 & 108.103 & 6.677.140,16 & 32,1 \\
\hline
\end{tabular}

A: Agendada / C: Confirmada / Abs: Absenteísmo / N: Absenteísmo total; Fonte Dados: SESA/ SISREG- Autores. 


\section{Discussão}

Historicamente, as políticas de saúde estimularam o crescimento do setor privado no Brasil e promoveram a privatização da assistência à saúde (Paim, Travassos, Almeida, Bahia, \& Macinko, 2011). Com recursos insuficientes, o SUS enfrenta inúmeros problemas ao longo da sua existência, colocando em risco o seu compromisso com a defesa do direito universal à saúde. Para suprir suas insuficiências, vem optando pela compra de serviços na gestão indireta, o que fortalece cada vez mais a ideologia da privatização (Paim, 2018). O termo privatização, aqui empregado, refere-se ao movimento de transferências de responsabilidades do setor público para o privado (Borges, Moimaz, Siqueira, \& Garbin, 2012).

As publicações cientificas sobre o tema são frágeis em relação às comparações entre os modelos de gestão presentes no SUS, com poucas teorias e fracos aportes metodológicos, inconclusivas quanto a superioridade de um modelo sobre o outro (Basu, Andrews, Kishore, Panjabi, \& Stuckler, 2012; Ravioli et al., 2018). Em sua maioria, os estudos carecem de avaliações econômicas para comparar duas ou mais alternativas, avaliando simultaneamente os custos e os resultados (La Forgia \& Harding, 2009). As análises comparativas ficam frágeis, considerando que o SUS não produz informações de custos nos seus serviços próprios de uma maneira geral e de forma sistemática, deixando assim lacunas não oferecendo subsídios mais concretos nas análises. Vale destacar, que o conceito de custos aqui aplicado refere-se à valoração dos recursos usados na produção de um bem ou serviço como recursos humanos, equipamentos, materiais de consumo, espaço físico, energia elétrica, água, entre outros (Vieira, 2017).

Nesse sentido, este estudo buscou preencher essa lacuna, propondo uma metodologia que agregasse mais informações para calcular o impacto financeiro na abordagem do absenteísmo como um fator de desperdício nas diferentes modalidades de gestão no SUS. Foi utilizado o mesmo banco de dados da pesquisa realizada nos anos de 2014 a 2016 , que encontrou para o absenteísmo a taxa média em consulta especializada de 38,6\% e para exames especializados a taxa de $32,1 \%$ (Beltrame, Oliveira, Santos, \& Santos Neto, 2019).

No entanto, ao levantar os dados administrativos disponíveis, constatou-se a limitação nas informações, restritas a valor dos procedimentos em sua maioria nos diferentes locais executantes. Assim, nos serviços próprios, o valor atribuído a cada procedimento foi o da Tabela SUS, por não haver centro de custos nos serviços. Ao utilizar essa tabela para efeito de cálculo, entendemos claramente que são valores que não traduzem a realidade do custo final do procedimento para a gestão pública. Observa-se a necessidade de aperfeiçoar a gestão de custos por procedimentos tornando a comparação mais fidedigna, fornecendo dados e subsídios para se atingir uma alocação eficiente dos recursos disponíveis na gestão dos serviços (Alemão, 2018). Ressalta-se que o desconhecimento dos custos é o maior indicador de ineficiência no provimento dos serviços públicos (Nunes, 1999).

Nesse estudo, utilizando-se, para calcular o impacto monetário com o absenteísmo, para os procedimentos de consulta e exames especializados somente a Tabela SUS (Tabela 2), como parâmetro de preço para todas as modalidades de gestão e usando a Tabela SUS e as complementares para os locais executantes que usam preços e custos diferenciados (Tabela 1), como os filantrópicos e público federal e com gestão de OSS, identificou-se uma diferença significativa no valor atribuído ao desperdício, sendo em consultas 38,4\% maior e em exames 124,8\% maior. Assim, ficam evidentes as diferenças nos percentuais usando as duas comparações. Considerando que a Tabela SUS pode estar defasada por não sofrer atualização dos seus preços nos últimos anos, pode-se sugerir que não supre as necessidades dos custos reais dos procedimentos e por isso, as contratações indiretas necessitam fazer o acréscimo de valor por procedimento usando tabela complementar. Levando em consideração os dados encontrados, fica evidenciado o aumento dos gastos na AE com as contratações na gestão indireta, reafirmando a importância e a necessidade da implantação da gestão dos custos no SUS, considerando que os recursos não são 
abundantes e o aumento de preços nessas negociações pode contribuir para o quadro, de modo a dificultar cada vez mais o acesso do usuário.

Ao analisar os resultados para a prestação de serviços em consultas especializadas, observou-se que a maior oferta compete ao setor público, enquanto os prestadores privados/filantrópicos/OSS são responsáveis por uma pequena porcentagem, estando em sintonia com a realidade do país (Romano \& Scatena, 2014; Solla \& Chioro, 2012). Considerando apenas o número de estabelecimentos e o que eles produzem, pode-se dizer que os prestadores filantrópicos são os que mais produzem tendo um papel complementar ao serviço público estadual, respeitando o que preceitua o artigo 199 da Constituição Federal. Entretanto, na prestação de serviços em exames especializados, a situação inverte: observa-se que a maioria da produção está nos prestadores do setor privado/filantrópico/OSS, e somente o mínimo no público. Os resultados demonstram que a contratação na administração indireta vem ganhando espaço maior na RSM-ES e que os investimentos em serviços públicos especializados são infinitamente menores, em consonância com dados do país (Romano \& Scatena, 2014). O serviço filantrópico passa a ser o prestador maior, seguido pelo público com gestão de OSS, o que evidencia que a oferta na atenção especializada para os exames ainda se encontra fortemente vinculada aos serviços da gestão indireta, colocando sob questionamento a complementaridade constitucional da iniciativa privada no SUS. Os interesses privados acabam determinando o padrão de oferta para o sistema, pois, de uma forma geral, os serviços públicos não possuem capacidade física instalada suficiente para suprir as necessidades na atenção especializada, se consideradas as diferentes demandas dos usuários (Solla \& Chioro, 2012).

Ainda analisando a oferta de procedimentos, os dados evidenciam que a administração indireta oferta menos em consultas, que são bem menos lucrativas, e ao contrário em exames especializados, sugerindo que esses prestadores tendem a selecionar casos e procedimentos de diagnóstico mais rentáveis, recusando outros de menor lucratividade (Oleskovicz et al., 2014). Em relação ao fato de a menor taxa de absenteísmo ser no serviço privado, destacam-se os relatos da gerência de regulação, segundo os quais estes serviços, para a realização de exames, têm um sistema mais efetivo de lembretes, mensagens e SMS para o usuário, que estimulam a uma frequência melhor, o que é confirmado na literatura em algumas pesquisas (GurolUrganci, de Jongh, Vodopivec-Jamsek, Atun, \& Car, 2013; Stubbs, Sanders, Jones, Geraci, \& Stephenson, 2012), quando analisado um sistema com e sem lembretes. Esta análise mostra que os lembretes de mensagens por telefone celular aumentam a frequência. O dado encontrado não é suficiente para se afirmar a vantagem da contratação privada, considerando que alguns estudos relevantes como o de Basu et al. (2012) que em revisão sistemática, comparou o desempenho do setor privado com o público, em países de renda média e baixa e os estudos avaliados não apoiaram a afirmação de que o setor privado é geralmente mais eficiente, responsável ou clinicamente mais eficaz do que o setor público.

Considerando as taxas altas e os valores desperdiçados na administração indireta, conforme Tabela 1, podemos afirmar que estão em discordância com os argumentos dos que defendem essas contratações para o SUS, alegando que são mais eficientes que a administração direta. Sabe-se que a contratação indireta suscita ampla controvérsia ideológica, política e econômica e jurídica, seja no Brasil ou no exterior (Coelho \& Greve, 2016). Os argumentos favoráveis às contratações desses serviços pelo SUS, principalmente das Organizações Sociais de Saúde, indicam que apresentam características que possibilitam maior agilidade à gestão, quando comparadas à administração direta, principalmente em relação às regras burocráticas. Sugerem que ampliam a capacidade de resposta rápida às demandas da saúde, ampliam a resolutividade para os usuários que conseguem ter acesso (Tibério, Souza, \& Sarti, 2010).

A resolutividade é questionável, quando se analisa os dados encontrados nesta pesquisa, considerando que o absenteísmo gera inúmeras consequências, podendo-se destacar prejuízos monetários (Bender et al., 2010; Ellis \& Jenkins, 2012; Gurol-Urganci et al., 2013; Jabalera Mesa et al., 2015), perda da eficiência da clínica e da gestão e atraso no tratamento adequado (Giunta et al., 2013). Segundo Travagin (2017), conforme preconiza a Lei Orgânica, as OSS não são complementares ao SUS, isto é, na 
prática são substitutivas, visto que, após a celebração do contrato de gestão, a parceria de fato é a transferência de atividades e recursos do público para o privado.

Com o objetivo de melhorar a gestão hospitalar em 2009, no Espírito Santo, iniciou-se a discussão sobre as OSS, em função de decreto estadual que regulamentava a Lei complementar estadual n. 489/2009, que criou o programa de OSS no estado. Surgem como instituições privadas sem fins lucrativos, estabelecem uma nova modalidade de relação entre o setor público e o privado por meio de contratos de gestão, com a finalidade de gerir os serviços de saúde com maior autonomia gerencial e sem os entraves burocráticos.

Nos contratos de gestão são estabelecidos compromissos acordados entre o Estado (nível central) e as OSS (nível local), com objetivos e metas (atendimentos, procedimentos e/ou outros indicadores) para um determinado período, no qual são estabelecidos percentuais de taxas de administração (Camargo, Prado, Bernardo, \& Lara, 2013). Em contrapartida, a administração central concede à local mais autonomia gerencial, liberando-a do controle dos meios, que passa a ser realizados sobre os resultados pactuados/alcançados (Ibañez \& Vecina Neto, 2007). Assim, em dezembro de 2009, foi firmado o primeiro contrato para a gestão do Hospital Estadual Central. No período da pesquisa, (2014 a 2016), existiam três hospitais com gestão de OSS para a RSM-ES. Os contratos disponibilizados no Portal da Transparência, no site on-line da SESA-ES, apontam valores a serem repassados significativos, mediante metas estabelecidas e resultados alcançados. Os custos atribuídos aos procedimentos, fornecidos pela SESA-ES, são muito maiores quando comparados aos preços na Tabela SUS e variam significativamente entre os estabelecimentos com gestão de OSS. Os valores monetários desperdiçados atribuídos ao absenteísmo nos exames especializados, são os maiores do grupo e muito expressivos.

A tarefa de regular e monitor nas administrações indiretas não é fácil para o gestor, pois existem algumas distorções importantes em serviços especializados, em destaque, quando não estão sujeitos aos mecanismos de regulação e controle (Solla \& Chioro, 2012). A SESA-ES admite ser um desafio, no âmbito das contratualizações na, pois nem todos os procedimentos ofertados estão regulados, sendo parte em autogestão do contratualizado. Considerando que o monitoramento às vezes é ausente e/ou incipiente, pode acontecer do local executante exceder o seu percentual de autogestão, chegando a ultrapassar o que foi contratualizado. Muitas vagas disponibilizadas pela regulação aos municípios são agendadas, mas os serviços não são executados por ausência do paciente, sendo essas vagas preenchidas com pacientes da autogestão, reforçando a necessidade de uma melhora no monitoramento (SESA, 2016). Mediante os dados disponibilizados no Portal da Transparência e da complexidade contábil para identificar valores pagos aos contratados, não foi possível aferir se o não produzido pelo absenteísmo não foi pago.

Assim, nessa dinâmica, muitas vagas não são utilizadas, prejudicando a continuidade do tratamento e ocasionando aumento da fila de espera nos municípios (Bender et al., 2010; Violin, 2007). Pode-se dizer que o absenteísmo é um tema complexo e precisa de ser analisado pelos gestores, considerando que é um fenômeno multicausal e que repercute em todos os envolvidos: gestão, trabalhador e usuário (Spedo, Pinto, \& Tanaka, 2010). A SESA-ES destaca como desafio o monitoramento, principalmente, nas instituições filantrópicas e nos públicos com gestão das OSS, que também operam na lógica da autogestão (SESA, 2016). Segundo Violin (2007), o processo de fiscalização da OSS ainda tem limitações, como a dificuldade de acesso às informações por parte dos órgãos de controle externo, como os tribunais de contas, e, mesmo internamente pelos órgãos de controle social do SUS.

Visando melhorar a gestão, o estado iniciou a regulação na RSM-ES em setembro de 2013 e os dados na pesquisa de 2014 a 2016, só foram possíveis devido à implantação do SISREG-ES. A regulação vem se aprimorando e tornando-se um importante instrumento de gestão. Pode ser entendida aqui como a capacidade de intervir nos processos de prestação de serviços, alterando ou orientando sua execução (Santos \& Merhy, 2006), com o objetivo de alcançar a eficiência, a equidade e o equilíbrio entre a oferta, demanda e financiamento, sendo um processo que precisa de aprimoramento para garantir qualidade 
na atenção à saúde (Vilarins, Shimizu, \& Gutierrez, 2012). Sob o prisma da equidade na assistência à saúde, pode-se inferir que a regulação é imprescindível e sua finalidade é assegurar que se atinjam os grandes objetivos sociais do sistema de saúde (Farias, Gurgel Jr., Costa, Brito, \& Buarque, 2011). Desse ponto de vista, Oliveira e Elias (Oliveira \& Elias, 2012), afirmam que a regulação tem sido uma resposta potencial para o enfrentamento de questões de diferentes naturezas nos sistemas de saúde, tais como a produção privada, o financiamento e a oferta de serviços de saúde. Dessa forma, é necessária uma regulação adequada por parte do Estado, para garantir que prevaleça o interesse público, independentemente da magnitude da participação privada no SUS.

Deve-se destacar também que a concentração dos serviços na capital Vitória e/ou em torno dela é semelhante ao que é observado em todo o país, as diversas modalidades de serviços que compõem o sistema de saúde não se distribuem uniformemente (Noronha, Noronha, Pereira, \& Costa, 2018). Os municípios menores não possuem demanda suficiente para abrigar todos os níveis de atenção à saúde em seu território, necessitando de serviços de outros municípios, para atender níveis complementares na atenção à saúde. Em termos de investimentos para se manterem serviços especializados em municípios pequenos com baixa demanda, não é viável, sendo um dos fatores que fortalece a necessidade da organização do sistema em redes de atenção com um sistema de regulação bem definido quanto às suas normas operacionais entre o estado e os municípios das regiões de saúde (Mendes, 2016).

A oferta de serviços na atenção especializada é um dos pontos de maior estrangulamento do SUS e um dos principais entraves para garantir a integralidade da atenção (Solla \& Chioro, 2012; Spedo et al., 2010). Segundo dados do Instituto de Pesquisa Econômica Aplicada (IPEA, 2011), que estudou a percepção dos usuários em relação aos serviços públicos, um dos principais problemas que apareceu em relação ao SUS, foi a demora para conseguir consulta na atenção especializada. Historicamente, esses serviços têm sido organizados e financiados com base na lógica de oferta de procedimentos, não priorizando as necessidades e o perfil epidemiológico da população (Solla \& Chioro, 2012).

É uma limitação do estudo a fragilidade do banco de dados sobre absenteísmo fornecidos pelo SISREG-ES, que apresenta falhas, uma vez que não é possível identificar se a falta ocorreu por problemas administrativos da gestão ou por ausência do usuário, ficando registrado somente como ausência do usuário. Outra limitação são as falhas recorrentes nos registros de atendimentos efetuados pelos locais executantes que não dão baixa no sistema, quando o usuário é atendido para confirmar, ficando assim, computados como absenteísmo procedimentos realizados, o que superestima os dados encontrados.

Ciente do problema, a SESA-ES adotou medidas administrativas para responder à necessidade de adequação do registro de todos os usuários atendidos, publicando a Portaria 084-R/2018, em 20/11/18, normatizando a baixa obrigatória diária no sistema dos atendimentos realizados, visando a obter dados reais sobre a atenção ao usuário. Assim, as taxas de absenteísmo encontradas e os respectivos valores monetários são questionáveis.

Por fim, em função das diferentes modalidades de gestão e as formas de desembolso monetário por serviços executados, principalmente nos filantrópicos e públicos com gestão de OSS, não foi possível identificar se o procedimento não realizado foi pago.

\section{Conclusão}

O absenteísmo na atenção especializada é crescente na RSM-ES, com prejuízos para a gestão pública e para os usuários, levando em consideração, principalmente a desassistência, podendo inviabilizar a ampliação da oferta de serviços especializados. Salienta-se que as taxas de absenteísmo e os valores monetários encontrados são expressivos, maiores nos serviços filantrópicos e com gestão de OSS e representam dados significativos para a gestão do sistema de saúde pública. Os dados monetários encontrados por modalidade são importantes informações que ajudam na reflexão para a tomada de decisão, 
sobre investimentos e prioridades nas contratações dos serviços da gestão administrativa indireta, auxiliando no processo de eficiência nos gastos em saúde. Considerando que estamos em um período crítico de investimentos no sistema público, há de se refletir sobre os custos pagos com os contratos principalmente na gestão por OSS, podendo inviabilizar a ampliação do acesso e da cobertura integral.

Contribuição importante para a discussão do tema, seriam pesquisas sobre as causas do absenteísmo relacionadas com a gestão e as particularidades do usuário, a fim de propor medidas viáveis de serem executadas, visando reduzi-lo, considerando a integralidade da atenção como um dos maiores desafios do SUS. O presente estudo tem suas limitações, entretanto, é o primeiro trabalho a apresentar dados sobre o tema no estado e evidencia e ressalta a importância de pesquisas avaliativas capazes de comparar as diferentes modalidades de gestão na assistência à saúde, destacando a importância de instituir informações sistematizadas dos custos dos bens e de serviços, para analisar seu desempenho e seus efeitos na qualidade da assistência prestada. Modernizar a gestão na saúde e otimizar os recursos disponíveis, combatendo o desperdício, sem comprometer a qualidade da assistência, será um desafio constante na agenda dos gestores do SUS na atualidade e nos próximos anos. Assim, pode-se concluir que é necessário o desenvolvimento de estudos na temática para ampliação do conhecimento e criação de estratégias para redução do absenteísmo e o desperdício de recursos.

\section{Agradecimentos}

O presente trabalho foi realizado com apoio da Coordenação de Aperfeiçoamento de Pessoal de Nível Superior Brasil (CAPES) - Código de Financiamento.

\section{Referências}

Alemão, M. M. (2018). A transposição da gestão de custos do setor privado para o público. Revista da Controladoria Geral da União, 10(17). Recuperado de https://ojs.cgu.gov.br/index.php/Revista_da_CGU/article/view/60/pdf_48.

Araujo, J. M. B. G. de., Maia, A. G., Moura, F. dos S. ., Duarte, M. P. M. ., Dantas, M. B. ., Oliveira, D. J. B. ., Nóbrega, J. C. da S., \& Oliveira, J. D. S. . (2021). The right to health and the role of the Unified Health System in times of pandemic in Brazil. Research, Society and Development, 10(11), e566101118005. https://doi.org/10.33448/rsd-v10i11.18005

Barreto, M. L., Teixeira, M. G., Bastos, F. I., Ximenes, R. A., Barata, R. B., \& Rodrigues, L. C. (2011). Successes and failures in the control of infectious diseases in Brazil: Social and environmental context, policies, interventions, and research needs. The Lancet, 377(9780), 1877-1889.

Basu, S., Andrews, J., Kishore, S., Panjabi, R., \& Stuckler, D. (2012). Comparative performance of private and public healthcare systems in low- and middleincome countries: A systematic review. PLoS medicine, 9(6), e1001244.

Beltrame, S. M., Oliveira, A. E., Santos, M. A. B. dos, \& Santos Neto, E. T. (2019). Absenteísmo de usuários como fator de desperdício: Desafio para sustentabilidade em sistema universal de saúde. Saúde em Debate, 43(123), 1015-1030.

Bender, A. D. S., Molina, L. R., \& Mello, A. L. S. F. D. (2010). Absenteísmo na Atenção Secundária e suas implicações na Atenção Básica, 11(2), 56-65.

Bittar, O. J. N., Magalhães, A., Felizola, N. B. G., \& Falcão, L. H. (2016). Absenteísmo em atendimento ambulatorial de especialidades no estado de São Paulo. Boletim Epidemiológico Paulista, 152(13), 19-32.

Borges, F. T., Moimaz, S. A. S., Siqueira, C. E., \& Garbin, C. A. S. (2012). Anatomia da privatização neoliberal do SUS: o papel das organizações sociais. São Paulo: Cultura Acadêmica.

Brasil. (2001).PORTARIA MS/GM N N 1606, DE 11 DE SETEMBRO DE 2001. http://bvsms.saude.gov.br/bvs/saudelegis/gm/2001/prt1606_11_09_2001.html

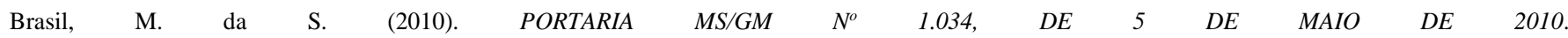
http://bvsms.saude.gov.br/bvs/saudelegis/gm/2010/prt1034_05_05_2010_rep.html

Camargo, T. A., Prado, O., Bernardo, R., \& Lara, A. C. H. S. (2013). Controle e transparência no modelo de organizações sociais de saúde do estado de São Paulo. VI Congresso CONSAD de Gestão Pública, Congresso Nacional de Saúde e Atenção Domiciliar. http://www.escoladegestao.pr.gov.br/arquivos/File/2013/V_CONSAD/VI_CONSAD/050.pdf

Cavalcanti, R. P., Cavalcanti, J. C. M., Serrano, R. M. S. M., \& Santana, P. R. D. (2013a). Absenteísmo de consultas especializadas nos sistemas de saúde público: Relação entre causas e o processo de trabalho de equipes de saúde da família, João Pessoa- PB, Brasil . Tempus- Actas de Saúde Coletiva, 7(2), 6384. 
Cavalcanti, R. P., Cavalcanti, J. C. M., Serrano, R. M. S. M., \& Santana, P. R. D. (2013b). Absenteísmo de consultas especializadas nos sistema de saúde público: Relação entre causas e o processo de trabalho de equipes de saúde da família, João Pessoa- PB, Brasil. Tempus- Actas de Saúde Coletiva, 7(2).

Coelho, V. S. P., \& Greve, J. (2016). As Organizações Sociais de Saúde e o Desempenho do SUS: Um Estudo sobre a Atenção Básica em São Paulo. Dados, 59(3), 867-901.

Dantas, L. F., Fleck, J. L., Cyrino Oliveira, F. L., \& Hamacher, S. (2018). No-shows in appointment scheduling - a systematic literature review. Health Policy, 122(4), 412-421.

Ellis, D. A., \& Jenkins, R. (2012). Weekday Affects Attendance Rate for Medical Appointments: Large-Scale Data Analysis and Implications. (T. Preis, Org.) PLOS ONE, 7(12), e51365.

Farias, S. F., Gurgel Jr., G. D., Costa, A. M., Brito, R. de L., \& Buarque, R. R. (2011). A regulação no setor público de saúde no Brasil: Os (des) caminhos da assistência médico-hospitalar. Ciência \& Saúde Coletiva, 16(suppl 1), 1043-1053.

Giovanella, L., Mendoza-Ruiz, A., Pilar, A. de C. A., Rosa, M. C. da, Martins, G. B., Santos, I. S., Silva, D. B., et al. (2018). Sistema universal de saúde e cobertura universal: Desvendando pressupostos e estratégias. Ciência \& Saúde Coletiva, 23(6), 1763-1776.

Giunta, D., Briatore, A., Baum, A., Luna, D., Waisman, G., \& Gonzalez Bernaldo de Quiros, F. (2013). Factors associated with nonattendance at clinical medicine scheduled outpatient appointments in a university general hospital. Patient Preference and Adherence, $1163-1170$.

Gurol-Urganci, I., de Jongh, T., Vodopivec-Jamsek, V., Atun, R., \& Car, J. (2013). Mobile phone messaging reminders for attendance at healthcare appointments. (Cochrane Consumers and Communication Group, Org.)Cochrane Database of Systematic Reviews. Recuperado fevereiro 20, 2019, de http://doi.wiley.com/10.1002/14651858.CD007458.pub3

Ibañez, N., \& Vecina Neto, G. (2007). Modelos de gestão e o SUS. Ciência \& Saúde Coletiva, 12, 1831-1840.

IPEA, I. de P. E. A. (2011). Retrato das desigualdades de gênero e raça (4º ed). Brasília, DF.

Jabalera Mesa, M. L., Morales Asencio, J. M., \& Rivas Ruiz, F. (2015). [Determinants and economic cost of patient absenteeism in outpatient departments of the Costa del Sol Health Agency]. Anales Del Sistema Sanitario De Navarra, 38(2), 235-245.

Kheirkhah, P., Feng, Q., Travis, L. M., Tavakoli-Tabasi, S., \& Sharafkhaneh, A. (2015). Prevalence, predictors and economic consequences of no-shows. BMC Health Services Research, 16(1). Recuperado fevereiro 14, 2019, de http://bmchealthservres.biomedcentral.com/articles/10.1186/s12913-015-1243-Z

La Forgia, G. M., \& Harding, A. (2009). Public-private partnerships and public hospital performance in São Paulo, Brazil. Health Affairs (Project Hope), 28(4), 1114-1126.

Mendes, E. V. (2016). O ACESSO À ATENÇÃO PRIMÁRIA À SAÚDE. CONASS - Conselho Nacional de Secretários de Saúde.

Noronha, J. C. de, Noronha, G. S. de, Pereira, T. R., \& Costa, A. M. (2018). Notas sobre o futuro do SUS: Breve exame de caminhos e descaminhos trilhados em um horizonte de incertezas e desalentos. Ciência \& Saúde Coletiva, 23(6), 2051-2059.

Nunes, M. A. (1999). Custos no serviço público. Revista do Serviço Público, 50(1), 37-63.

Ocké-Reis, C. O. (2016). SUS: O desafio de ser único. Rio de Janeiro, RJ: Editora Fiocruz.

Oleskovicz, M., Oliva, F. L., Grisi, C. C. de H. e, Lima, A. C., \& Custódio, I. (2014). [Overbooking in an outpatient healthcare facility in the Brazilian Unified National Health System]. Cadernos De Saude Publica, 30(5), 1009-1017.

Oliveira, R. R. de, \& Elias, P. E. M. (2012). Conceitos de regulação em saúde no Brasil. Revista de Saúde Pública, 46(3), 571-576.

Paim, J. S. (2009). O que é o SUS. Fiocruz.

Paim, J. S. (2018). Sistema Único de Saúde (SUS) aos 30 anos. Ciência \& Saúde Coletiva, 23(6), 1723-1728.

Paim, J., Travassos, C., Almeida, C., Bahia, L., \& Macinko, J. (2011). The Brazilian health system: History, advances, and challenges. The Lancet, 377(9779), $1778-1797$.

Ravioli, A. F., Soárez, P. C. D., \& Scheffer, M. C. (2018). Modalidades de gestão de serviços no Sistema Único de Saúde: Revisão narrativa da produção científica da Saúde Coletiva no Brasil (2005-2016). Cadernos de Saúde Pública, 34(4). http://www.scielo.br/scielo.php?script=sci_arttext\&pid=S0102311 X2018000402001\&lng=pt\&tlng=pt

REBELlO, R. (2015, julho 20). Faltas em consultas médicas pelo SUS chegam a $46 \%$ no ESComunidade de Práticas. https://blog.atencaobasica.org.br/2015/09/14/faltas-em-consultas-medicas-pelo-sus-chegam-a-46-no-es/

Romano, C. M. C., \& Scatena, J. H. G. (2014). A relação público-privada no SUS: Um olhar sobre a assistência ambulatorial de média e alta complexidade na região de saúde da Baixada Cuiabana em Mato Grosso. Revista de Administração Pública, 48(2), 439-458.

Santos, F. P. dos, \& Merhy, E. E. (2006). A regulação pública da saúde no Estado brasileiro: Uma revisão. Interface-Comunicação, Saúde, Educação, $10(19), 25-41$.

Schmidt, M. I., Duncan, B. B., e Silva, G. A., Menezes, A. M., Monteiro, C. A., Barreto, S. M., Chor, D., et al. (2011). Chronic non-communicable diseases in Brazil: Burden and current challenges. The Lancet, 377(9781), 1949-1961.

Secchi, L. (2009). Modelos organizacionais e reformas da administração pública. Revista de Administração Pública, 43(2), 347-369. 
Research, Society and Development, v. 11, n. 1, e31011124797, 2022

(CC BY 4.0) | ISSN 2525-3409 | DOI: http://dx.doi.org/10.33448/rsd-v11i1.24797

SESA. (2011). Plano Diretor de Regionalização - PDR - ES 2011. Recuperado de https://saude.es.gov.br/Media/sesa/SISPACTO/PDRPlano\%20Diretor\%20de\%20Regionaliza\%C3\%A7\%C3\%A3o_ES_2011.pdf

SESA, E. (2016). Plano de Intervenção Regional da Região de Saúde Metropolitana do ES.

Solla, J., \& Chioro, A. (2012). Atenção ambulatorial especializada. In: GIOVANELLA, Lígia et al. (Org.). Políticas e sistemas de saúde no Brasil. (2º ed). RIO DE JANEIRO: Fiocruz.

Spedo, S. M., Pinto, N. R. da S., \& Tanaka, O. Y. (2010). O difícil acesso a serviços de média complexidade do SUS: O caso da cidade de São Paulo, Brasil. Physis: Revista de Saúde Coletiva, 20(3), 953-972.

Stubbs, N. D., Sanders, S., Jones, D. B., Geraci, S. A., \& Stephenson, P. L. (2012). Methods to Reduce Outpatient Non-attendance. The American Journal of the Medical Sciences, 344(3), 211-219.

Tesser, C. D., \& Poli Neto, P. (2017). Atenção especializada ambulatorial no Sistema Único de Saúde: Para superar um vazio. Ciência \& Saúde Coletiva, 22(3), 941-951.

Tibério, A. A., Souza, E. M., \& Sarti, F. M. (2010). Considerações sobre avaliação de estabelecimentos de saúde sob gestão de OSS: O caso do Hospital Geral do Grajaú. Saúde e Sociedade, 19(3), 557-568.

Travagin, L. B. (2017). O avanço do capital na saúde: Um olhar crítico às Organizações Sociais de Saúde. Saúde em Debate, 41(115), 995-1006.

Vieira, F. S. (2017). Produção de informações de custos para a tomada de decisão no Sistema Único de Saúde:uma questão para a política pública. IPEA Texto para discussão, (2314).

Vilarins, G. C. M., Shimizu, H. E., \& Gutierrez, M. M. U. (2012). A regulação em saúde: Aspectos conceituais e operacionais. Saúde em Debate, 36(95), 640647.

Violin, T. C. (2007). Da administração pública patrimonialista à administração pública gerencial e a burocracia. Rev Adm Const., 7(30), 225-47. 